\title{
Serum ferritin as an independent predictor of non- alcohol fatty liver disease in general adults: the TCLSIH cohort study
}

\section{Yeqing Gu}

Chinese Academy of Medical Sciences \& Peking Union Medical College Institute of Radiation Medicine

\section{Ge Meng}

Tianjin Medical University

\section{Xiaowei Chen}

Tianjin Medical University

Jinhan Wang

Chinese Academy of Medical Sciences \& Peking Union Medical College Institute of Radiation Medicine

Qing Zhang

Tianjin Medical University General Hospital

\section{Li Liu}

Tianjin Medical University General Hospital

\section{Hongmei Wu}

Tianjin Medical University

\section{Shunming Zhang}

Tianjin Medical University

\section{Tingjing Zhang}

Tianjin Medical University

\section{Xuena Wang}

Tianjin Medical University

\section{Juanjuan Zhang}

Tianjin Medical University

\section{Shaomei Sun}

Tianjin Medical University General Hospital

\section{Xing Wang}

Tianjin Medical University General Hospital

\section{Qiyu Jia}

Tianjin Medical University General Hospital

\section{Kun Song}

Tianjin Medical University General Hospital

\section{Qiang Liu}


Chinese Academy of Medical Sciences \& Peking Union Medical College Institute of Radiation Medicine Kaijun Niu ( $\square$ nkj0809@gmail.com )

Tianjin Medical University https://orcid.org/0000-0002-8772-2481

\section{Research Article}

Keywords: Serum ferritin, Non-alcoholic fatty liver disease, Incidence, Abdominal ultrasonography, Early detection, Screen, Adult population, Cohort, Prospective study, Epidemiology

Posted Date: February 21st, 2022

DOI: https://doi.org/10.21203/rs.3.rs-1321357/v1

License: (9) This work is licensed under a Creative Commons Attribution 4.0 International License. Read Full License 


\section{Abstract \\ Background}

Serum ferritin is an important iron storage protein in the human body, which indicates iron storage and the body's nutritional status, and is associated with a variety of chronic diseases. Few prospective studies have assessed the association between serum ferritin levels and non-alcoholic fatty liver disease (NAFLD). This prospective cohort study aimed to explore whether serum ferritin levels were associated with the incidence of NAFLD in a large-scale adult population.

\section{Methods}

A total of 15,748 participants without baseline NAFLD were enrolled in the cohort study (1-9 years followup periods, median: 4.5 years). Serum ferritin levels were determined by protein chip-chemiluminescence method, and NAFLD was diagnosed by abdominal ultrasonography. Multivariable Cox proportional hazards regression models were used to assess the association between baseline serum ferritin levels and the risk of NAFLD.

\section{Results}

During the follow-up period, 3,374 new NAFLD cases were observed, and the incidence rate of NAFLD was 83.4 per 1000 person-years. In the fully adjusted model, the hazards ratios ( $95 \%$ confidence interval) for incident NAFLD across serum ferritin levels quartiles were 1.06 (0.94-1.19), 1.25 (1.11-1.41), and 1.52 (1.28-1.79) ( $P$ for trend $<0.0001)$. Receiver operating characteristic $(R O C)$ analysis showed that the optimal cut-off values were $55.9 \mathrm{mg} / \mathrm{dl}$ for serum ferritin levels (Area under the ROC curve (AUC) $=0.61$ )

\section{Conclusion}

Increased serum ferritin level was independently associated with a higher risk of incident NAFLD in a large-scale general population. It is suggested that serum ferritin levels can be useful for the early detection of subjects at high risk of developing NAFLD.

\section{Introduction}

Non-alcoholic fatty liver disease (NAFLD) is characterized by an abnormal accumulation of fat in liver cells and has been a major public health issue worldwide [1]. In recent years, NAFLD has emerged as the most common chronic liver disease, affecting $25 \%$ of the global population [2, 3]. The prevalence of NAFLD in Asia ranges from 15-40\% [4]. A recent meta-analysis in China showed that the national prevalence of NAFLD was $29.2 \%$ and revealed that China experienced an unexpected rapid increase in cases of NAFLD over a short period [5]. NAFLD is associated with liver-related morbidity and mortality 
and increases the risk of important extrahepatic chronic diseases, such as cardiovascular disease (CVD), diabetes, and chronic kidney disease [6]. Therefore, searching for effective prevention strategies are needed to control the development of NAFLD.

Serum ferritin is an important iron storage protein in the human body, which indicates iron storage and body nutritional status, and is associated with a variety of diseases [7]. Previous experiments studies have reported that diet-induced hepatic iron overload can increase hepatic free fatty acid (FFA) content and insulin resistance [8]. Hepatic iron overload also worsens mitochondrial dysfunction in the liver in a rat model of iron overload [9]. Moreover, excessive iron levels lead to increased oxidative stress [10]. Fatty acid accumulation, insulin resistance, mitochondrial dysfunction, and oxidative stress can lead to the development of NAFLD [11]. These reports prompted us to hypothesize that body iron stores as reflected by serum ferritin levels may be a strong predictor for the risk of NAFLD.

To our knowledge, only one cohort study conducted in middle-aged healthy Korean men $(n=2,410)$ found that serum ferritin level was an independent risk factor for incidence of NAFLD [12]. However, few studies have assessed the association between serum ferritin level and incidence of NAFLD in the general adult population. Therefore, we conducted a prospective cohort to explore whether serum ferritin level was an independent predictive factor for the incidence of NAFLD among the large-scale general adult population in China.

\section{Methods}

\section{Study population}

The Tianjin Chronic Low-grade Systemic Inflammation and Health (TCLSIH) cohort study is a dynamic prospective cohort study and the details of the cohort study have been described elsewhere [13]. In brief, the study participants were randomly recruited between January 2010 and December 2019 from the general population who took part in annual health checkups in Tianjin, China. The study protocol was approved by The Institutional Review Board of Tianjin Medical University. All participants provided written informed consent.

Figure 1 showed the flowchart for the selection of the study population. A total of 31,821 participants received at least one health examinations, including blood tests and liver ultrasonography. To be eligible for the present study, we included participants with alcoholic fatty liver disease $(n=1,010)$, other liver diseases $(n=209)$, CVD $(n=1,508)$, and cancer $(n=230)$ at baseline. Moreover, baseline NAFLD $(n=$ $10,887,37.7 \%$ ) was excluded. Finally, participants who did not undergo health examinations during follow-up were excluded $(n=2,229)$.

\section{Assessment of serum ferritin levels}

Fasting blood samples were taken in the morning after a 12-h overnight fast from venipuncture of the cubital vein. Serum ferritin levels were measured using the Quantitative Kit for Tumor Markers (Huzhou 
Shukang Biological Technology) by the protein chip-chemiluminescence method and expressed as $\mathrm{ng} / \mathrm{mL}$. The measurement range of the assay was $5-600 \mathrm{ng} / \mathrm{ml}$. The coefficients of variation of intraand inter-assay were less than $15 \%$. In the present analysis, serum ferritin levels were divided into four groups (quartiles).

\section{Assessment of NAFLD}

Fatty liver disease (FLD) was tested by abdominal ultrasonography, which was carried out by experienced sonographers using a TOSHIBA SSA-660A ultrasound machine (Toshiba, Tokyo, Japan), with a 2-5 MHz curved array probe. Images were also assessed by an experienced hepatologist. FLD was diagnosed if participants had two or more abnormal findings of liver ultrasonography as follows: diffusely increased echogenicity liver, liver echogenicity greater than kidney or spleen, or vascular blurring and the gradual attenuation of ultrasound signal. NAFLD was diagnosed without a history of heavy alcohol drinking ( $>210 \mathrm{~g} /$ week in males and $>140 \mathrm{~g} /$ week in females) [14] on the basis of the presence of fatty liver. Abdominal ultrasonography was conducted every year, and NAFLD was diagnosed yearly during followup.

\section{Assessment of covariates}

Sociodemographic characteristics (age and sex), lifestyle factors (smoking and alcohol drinking status), and personal and family disease history were obtained by using a detailed and constructed questionnaire. Body height $(\mathrm{m})$, weight $(\mathrm{kg})$, and waist circumference $(\mathrm{WC})(\mathrm{cm})$ were measured during annual health checkups by trained nurses. Body mass index (BMI) calculated from anthropometric measurements $\left(\mathrm{kg} / \mathrm{m}^{2}\right)$.

Blood tests including total cholesterol (TC), low-density lipoprotein cholesterol (LDL-C), high-density lipoprotein cholesterol (HDL-C), triglycerides (TG), fasting blood glucose (FBG), alanine aminotransferase (ALT), aspartate transaminase (AST), gamma-glutamyl transpeptidase (GGT), and hemoglobin (Hb), which were measured from fasting blood samples. Diabetes was defined as fasting blood glucose $\geq 7.0$ $\mathrm{mmol} / \mathrm{L}$ or a self-reported history of diabetes [15]. Hyperlipidemia was defined as total cholesterol $\geq 5.17$ $\mathrm{mmol} / \mathrm{L}$, triglycerides $\geq 1.7 \mathrm{mmol} / \mathrm{L}$, or low-density lipoprotein cholesterol $\geq 3.37 \mathrm{mmol} / \mathrm{L}$, or taking lipidlowering drugs [16]. Anemia was defined as $\mathrm{Hb}<120 \mathrm{~g} / \mathrm{L}$ in nonpregnant adult females and $<130 \mathrm{~g} / \mathrm{L}$ in males [17]. Blood pressure (BP) was measured 2 times using the TM-2655 oscillometric device (A\&D) and estimates from the 2 measurements were averaged. Hypertension was defined as systolic blood pressure (SBP) $\geq 140 \mathrm{~mm} \mathrm{Hg}$ or diastolic blood pressure (DBP) $\geq 90 \mathrm{~mm} \mathrm{Hg}$ or having a history of hypertension [18].

\section{Statistical analysis}

The distribution of all continuous variables was checked by one-sample Kolmogorov-Smirnov test $(n \geq 2,000)$. For the baseline participants characteristics, descriptive data were expressed as geometric mean $(95 \%$ confidence interval, $95 \% \mathrm{Cl}$ ) for continuous variables due to skewed distribution 
and percentages for categorical variables. The differences among the incident NAFLD categories were examined by using analysis of covariance (ANCOVA) for continuous variables, and multiple logistic regression analysis for proportional variables after adjustment for age and sex.

The Cox proportional hazards regression models were used to assess the association between serum ferritin levels and the incidence of NAFLD. Three multivariable models were fitted in our analyses. Model 1 was a crude model. Model 2 was adjusted for baseline age (continuous variable), sex (males or females), and BMI continuous variable). Model 3 was further adjusted for baseline serum ferritin, hypertension (yes or no), hyperlipidemia (yes or no), diabetes (yes or no), anemia (yes or no), smoking status (current, former, or never), alcohol drinking status (everyday drinker, sometime drinker, ex-drinker, or non-drinker), family history of diseases (including cardiovascular disease, hypertension, hyperlipidemia, and diabetes [each yes or no]). Hazard ratios (HRs) and 95\% $\mathrm{Cl}$ were calculated. Multicollinearity among the covariables in the fully adjusted models was diagnosed using variance inflation factor, and the results showed that collinearity was acceptable (all variance inflation factors are less than 10 ).

We stratified the participants by potential effect modifiers including age ( $<50$ or $\geq 50$ years), sex (males or females), BMI ( $<24$ or $\geq 24 \mathrm{~kg} / \mathrm{m}^{2}$ ), smoking status (current, former, or never), alcohol drinking status (everyday drinker, sometime drinker, ex-drinker, or non-drinker), hypertension (yes or no), hyperlipidemia (yes or no), diabetes (yes or no), anemia (yes or no) and then examined the interactions between these variables and serum ferritin levels.

To assess the robustness of the results, we performed a sensitivity analysis by excluding participants diagnosed with NAFLD for less than one year from baseline survey. Moreover, since anemia was associated with serum ferritin level, we further performed a sensitivity analysis after excluding participants with anemia.

Receiver operating characteristic (ROC) curves were used to assess the ability of the serum ferritin levels in diagnosing NAFLD. The optimal cut-off points used were the peaks of the curve, where the sum of sensitivity and specificity is at maximum.

All statistical analyses were performed using SAS software, version 9.3 (SAS Institute Inc., Cary, NC, USA). All tests were two-sided, and $P<0.05$ was considered statistically significant.

\section{Results}

A total of 15,748 participants were enrolled in the final analysis. The mean (standard deviation) age was 44.5 (12.0) years, and the proportion of males was 45.5\%. 3,374 new NAFLD cases were observed, and the incidence rate of NAFLD was 83.4 per 1000 person-years across the 9-year follow-up period (range, 1 to 9 years; median, 4.5 years).

Table 1 showed the age and sex-adjusted baseline characteristics associated with incident NAFLD. Compared with subjects who have not incident NAFLD, those participants with incident NAFLD tended to 
have a higher age, BMI, WC, TC, LDL-C, TG, SBP, DBP, FBG, ALT, AST, GGT, serum ferritin, and a higher proportion of males, proportion of hypertension, diabetes, and hyperlipidemia, proportion of smokers, proportion with a family history of CVD, hypertension, and diabetes and lower HDL-C and lower proportion of non-smokers (all $P$ values $<0.05$ ). No significant differences were observed for other factors (all $P>0.05)$. 
Table 1

Age- and sex-adjusted baseline participant characteristics by status of $\operatorname{NAFLD~}(n=15,748)$ a.

\section{NAFLD status}

$P$ value $^{\text {b }}$

No Yes

No. of subjects

Age $(y)$

Sex (male, \%)

$\operatorname{BMI}\left(\mathrm{kg} / \mathrm{m}^{2}\right)$

Waist circumference $(\mathrm{cm})$

$\mathrm{TC}(\mathrm{mmol} / \mathrm{L})$

LDL-C (mmol/L)

HDL-C (mmol/L)

TG (mmol/L)

$\mathrm{SBP}(\mathrm{mmHg})$

$\operatorname{DBP}(\mathrm{mmHg})$

FBG (mmol/L)

ALT (mmol/L)

AST (mmol/L)

GGT (mmol/L)

Serum ferritin $(\mathrm{ng} / \mathrm{mL})$

Hypertension (\%)

Diabetes (\%)

Hyperlipidemia (\%)

Anemia (\%)

Smoking status (\%)

Smoker

Ex-smoker

Non-smoker

Drinker (\%)
12,374

$42.3(42.1,42.5)^{c}$

40.7

$22.8(22.7,22.8)$

$78.4(78.2,78.5)$

$4.73(4.72,4.75)$

$2.68(2.66,2.69)$

$1.46(1.45,1.46)$

$0.97(0.96,0.97)$

$115.8(115.5,116.1)$

$73.3(73.1,73.5)$

$4.89(4.88,4.90)$

$14.5(14.4,14.6)$

$17.3(17.1,17.4)$

$18.3(18.1,18.6)$

$75.5(74.7,76.4)$

16.2

2.30

39.3

7.68

15.6

26.3

5.75

67.9

28.0

4.21

57.1

3.26

81.1
$<0.0001$

$<0.0001$

$24.5(24.4,24.6) \quad<0.0001$

$82.5(82.2,82.7) \quad<0.0001$

$4.85(4.82,4.88) \quad<0.0001$

$2.82(2.79,2.84) \quad<0.0001$

$1.33(1.32,1.34) \quad<0.0001$

$1.22(1.20,1.23) \quad<0.0001$

$118.7(118.2,119.2) \quad<0.0001$

$75.5(75.2,75.9) \quad<0.0001$

$4.94(4.92,4.96) \quad<0.001$

$17.0(16.7,17.2) \quad<0.0001$

$17.6(17.3,17.9) \quad 0.02$

$22.8(22.2,23.4)$

$<0.0001$

$79.5(77.9,81.2)$

$<0.0001$

$<0.0001$

$<0.01$

$<0.0001$

$\begin{array}{ll}4.71 & 0.14\end{array}$ 


\begin{tabular}{|c|c|c|c|}
\hline & \multicolumn{2}{|c|}{ NAFLD status } & \multirow[t]{2}{*}{$P$ value ${ }^{\text {b }}$} \\
\hline & No & Yes & \\
\hline Everyday & 2.62 & 4.03 & 0.81 \\
\hline Sometime & 48.3 & 56.6 & 0.21 \\
\hline Ex-drinker & 6.57 & 6.17 & 0.77 \\
\hline Non-drinker & 42.5 & 33.2 & 0.20 \\
\hline \multicolumn{4}{|c|}{ Family history of diseases (\%) } \\
\hline CVD & 32.2 & 37.5 & $<0.0001$ \\
\hline Hypertension & 50.4 & 56.2 & $<0.0001$ \\
\hline Hyperlipidemia & 0.36 & 0.50 & 0.23 \\
\hline Diabetes & 23.0 & 26.9 & $<0.0001$ \\
\hline \multicolumn{4}{|c|}{$\begin{array}{l}\text { a NAFLD, non-alcoholic fatty liver disease; BMI, body mass index; TC, total cholesterol; LDL-C, low } \\
\text { density lipoprotein cholesterol; HDL-C, high-density lipoprotein-cholesterol; TG, triglycerides; SBP, } \\
\text { systolic blood pressure; DBP, diastolic blood pressure; FBG, fast blood glucose; ALT, alanine } \\
\text { aminotransferase; AST, aspartate transaminase; GGT, gamma-glutamyl transpeptidase; CVD, } \\
\text { cardiovascular diseases. }\end{array}$} \\
\hline \multicolumn{4}{|c|}{ b Analysis of covariance or logistic regression analysis. } \\
\hline
\end{tabular}

The unadjusted and adjusted associations between baseline serum ferritin levels and the incidence of NAFLD were shown in Table 2. In the fully adjusted model, a positive and significant trend across serum ferritin levels quartiles was observed for the incidence of NAFLD, and the HRs $(95 \% \mathrm{Cl})$ for incident NAFLD across serum ferritin levels quartiles were $1.06(0.94,1.19), 1.25(1.11,1.41)$, and $1.52(1.28,1.79)$ ( $P$ for trend $<0.0001$ ). 
Table 2. Adjusted relationships of baseline serum ferritin levels to incident NAFLD $(n=15,748)$.

\begin{tabular}{|c|c|c|c|c|c|}
\hline & \multicolumn{4}{|c|}{ Quartiles of ferritin } & \multirow{2}{*}{$\begin{array}{l}P \text { for } \\
\text { trend }^{\text {a }}\end{array}$} \\
\hline & Level 1 & Level 2 & Level 3 & Level 4 & \\
\hline $\begin{array}{l}\text { Serum ferritin levels }(\mathrm{ng} / \mathrm{mL} \text {, } \\
\text { range) }\end{array}$ & $0 \sim 45$ & $45 \sim 65$ & $65 \sim 129$ & $129 \sim 1103$ & - \\
\hline No. of subjects & 3,938 & 3,936 & 3,937 & 3,937 & - \\
\hline Person-years of follow up & 11,375 & 10,965 & 9,835 & 8,285 & - \\
\hline No. of NAFLD & 581 & 639 & 969 & 1,185 & - \\
\hline Model $1^{b}$ & reference & $\begin{array}{l}1.14(1.02, \\
1.27)^{\mathrm{c}}\end{array}$ & $\begin{array}{l}1.93(1.74, \\
2.14)\end{array}$ & $\begin{array}{l}2.80(2.53, \\
3.09)\end{array}$ & $<0.0001$ \\
\hline Model $2^{d}$ & reference & $\begin{array}{l}1.07(0.96, \\
1.20)\end{array}$ & $\begin{array}{l}1.30(1.16, \\
1.46)\end{array}$ & $\begin{array}{l}1.62(1.44 \\
1.83)\end{array}$ & $<0.0001$ \\
\hline Model $3^{e}$ & reference & $\begin{array}{l}1.06(0.94 \\
1.19)\end{array}$ & $\begin{array}{l}1.25(1.11, \\
1.41)\end{array}$ & $\begin{array}{l}1.52(1.28 \\
1.79)\end{array}$ & $<0.0001$ \\
\hline
\end{tabular}

NAFLD, non-alcohol fatty liver disease.

${ }^{a}$ Analysis by Cox proportional hazards regression model.

${ }^{\mathrm{b}}$ Crude model.

${ }^{c}$ Adjusted hazard ratios (95\% confidence interval) (all such values).

${ }^{\mathrm{d}}$ Adjusted for age, sex, and baseline body mass index.

e Additionally adjusted for baseline serum ferritin, hypertension, diabetes, hyperlipidemia, anemia, smoking status, alcohol drinking status, and family history of diseases (hypertension, cardiovascular disease, hyperlipidemia and diabetes).

After excluding participants diagnosed with NAFLD for less than one year from baseline survey $(\mathrm{n}=$ $2,374)$, similar associations between serum ferritin levels and incident NAFLD were observed, and the HRs $(95 \% \mathrm{Cl})$ for incident NAFLD across serum ferritin levels quartiles were $1.00(0.80,1.25), 1.57(1.26,1.96)$, and $2.07(1.55,2.76)$ ( $P$ for trend $<0.0001)$ (Supplemental Table 1). After excluding participants with anemia $(n=1,109)$, the associations between serum ferritin levels and incident NAFLD did not change (data not shown).

Table 3 showed the associations between serum ferritin levels and risk of incident NAFLD stratified by major covariates, including age, sex, BMI, smoking status, alcohol drinking status, hypertension, hyperlipidemia, diabetes, and anemia. The results showed significant interactions between serum ferritin levels and BMI, hypertension, hyperlipidemia, and anemia for incident NAFLD in the final models ( $P$ for 
interaction $<0.05)$. No evidence showed that there were interactions between serum ferritin levels and other potential confounder factors for incident NAFLD (all $P$ for interaction $>0.18$ ).

Table 3. Associations between serum ferritin levels and risk of incident NAFLD stratified by major covariates $^{a}$. 


\begin{tabular}{|c|c|c|c|c|c|c|}
\hline \multirow[t]{2}{*}{ Covariates } & \multicolumn{4}{|c|}{ Quartiles of ferritin } & \multirow{2}{*}{$\begin{array}{l}P \text { for } \\
\text { trend }^{b}\end{array}$} & \multirow{2}{*}{$\begin{array}{l}P \text { for } \\
\text { interaction }\end{array}$} \\
\hline & Level 1 & Level 2 & Level 3 & Level 4 & & \\
\hline \multicolumn{7}{|l|}{ Age (years) } \\
\hline$\geq 50$ & reference & $\begin{array}{l}1.15(0.98, \\
1.35)^{c}\end{array}$ & $\begin{array}{l}1.28(1.09, \\
1.51)\end{array}$ & $\begin{array}{l}1.40(1.18, \\
1.66)\end{array}$ & $<0.0001$ & \multirow[t]{2}{*}{0.60} \\
\hline$<50$ & reference & $\begin{array}{l}1.18(1.02, \\
1.38)\end{array}$ & $\begin{array}{l}1.19(1.01, \\
1.41)\end{array}$ & $\begin{array}{l}1.57(1.32, \\
1.86)\end{array}$ & $<0.0001$ & \\
\hline \multicolumn{7}{|l|}{ Sex } \\
\hline Males & reference & $\begin{array}{l}1.08(0.96, \\
1.23)\end{array}$ & $\begin{array}{l}1.38(1.22, \\
1.56)\end{array}$ & $\begin{array}{l}1.40(1.24, \\
1.58)\end{array}$ & $<0.0001$ & \multirow[t]{2}{*}{0.82} \\
\hline Females & reference & $\begin{array}{l}1.08(0.91, \\
1.28)\end{array}$ & $\begin{array}{l}1.11(0.94, \\
1.31)\end{array}$ & $\begin{array}{l}1.42(1.21, \\
1.66)\end{array}$ & $<0.0001$ & \\
\hline \multicolumn{7}{|l|}{ BMI $\left(\mathrm{kg} / \mathrm{m}^{2}\right)$} \\
\hline$\geq 24.0$ & reference & $\begin{array}{l}1.06(0.93, \\
1.21)\end{array}$ & $\begin{array}{l}1.25(1.08 \\
1.44)\end{array}$ & $\begin{array}{l}1.38(1.19, \\
1.60)\end{array}$ & $<0.0001$ & \multirow[t]{2}{*}{$<0.0001$} \\
\hline$<24.0$ & reference & $\begin{array}{l}1.16(0.96, \\
1.40)\end{array}$ & $\begin{array}{l}1.47(1.22, \\
1.76)\end{array}$ & $\begin{array}{l}1.73(1.43, \\
2.11)\end{array}$ & $<0.0001$ & \\
\hline \multicolumn{7}{|l|}{ Smoking status } \\
\hline Current smoker & reference & $\begin{array}{l}1.12(0.92, \\
1.37)\end{array}$ & $\begin{array}{l}1.25(1.02 \\
1.53)\end{array}$ & $\begin{array}{l}1.34(1.09, \\
1.65)\end{array}$ & $<0.01$ & \multirow[t]{3}{*}{0.18} \\
\hline Ex-smoker & reference & $\begin{array}{l}1.24(0.90, \\
1.69)\end{array}$ & $\begin{array}{l}1.41(1.03, \\
1.94)\end{array}$ & $\begin{array}{l}1.76(1.27, \\
2.43)\end{array}$ & $<0.001$ & \\
\hline Non-smoker & reference & $\begin{array}{l}1.09(0.95 \\
1.24)\end{array}$ & $\begin{array}{l}1.28(1.13 \\
1.46)\end{array}$ & $\begin{array}{l}1.60(1.39, \\
1.84)\end{array}$ & $<0.0001$ & \\
\hline \multicolumn{7}{|l|}{$\begin{array}{l}\text { Alcohol drinking } \\
\text { status }\end{array}$} \\
\hline Everyday & reference & $\begin{array}{l}1.07(0.93, \\
1.23)\end{array}$ & $\begin{array}{l}1.30(1.12, \\
1.49)\end{array}$ & $\begin{array}{l}1.50(1.29, \\
1.73)\end{array}$ & $<0.0001$ & \multirow[t]{4}{*}{0.71} \\
\hline Sometime & reference & $\begin{array}{l}1.05(0.91, \\
1.21)\end{array}$ & $\begin{array}{l}1.29(1.11, \\
1.49)\end{array}$ & $\begin{array}{l}1.49(1.29, \\
1.73)\end{array}$ & $<0.0001$ & \\
\hline Ex-drinker & reference & $\begin{array}{l}1.02(0.89 \\
1.17)\end{array}$ & $\begin{array}{l}1.25(1.08 \\
1.43)\end{array}$ & $\begin{array}{l}1.44(1.25, \\
1.66)\end{array}$ & $<0.0001$ & \\
\hline Non-drinker & reference & $\begin{array}{l}1.07(0.95, \\
1.21)\end{array}$ & $\begin{array}{l}1.29(1.14, \\
1.45)\end{array}$ & $\begin{array}{l}1.57(1.39, \\
1.78)\end{array}$ & $<0.0001$ & \\
\hline
\end{tabular}




\begin{tabular}{|c|c|c|c|c|c|c|}
\hline \multirow[t]{2}{*}{ Covariates } & \multicolumn{4}{|c|}{ Quartiles of ferritin } & \multirow{2}{*}{$\begin{array}{l}P \text { for } \\
\text { trend }\end{array}$} & \multirow{2}{*}{$\begin{array}{l}P \text { for } \\
\text { interaction }\end{array}$} \\
\hline & Level 1 & Level 2 & Level 3 & Level 4 & & \\
\hline Yes & reference & $\begin{array}{l}1.15(0.94 \\
1.40)\end{array}$ & $\begin{array}{l}1.13(0.91 \\
1.39)\end{array}$ & $\begin{array}{l}1.21(0.98, \\
1.50)\end{array}$ & 0.12 & $<0.001$ \\
\hline No & reference & $\begin{array}{l}1.08(0.94 \\
1.24)\end{array}$ & $\begin{array}{l}1.32(1.15 \\
1.51)\end{array}$ & $\begin{array}{l}1.65(1.43, \\
1.91)\end{array}$ & $<0.0001$ & \\
\hline \multicolumn{7}{|c|}{ Hyperlipidemia } \\
\hline Yes & reference & $\begin{array}{l}1.05(0.91, \\
1.21)\end{array}$ & $\begin{array}{l}1.24(1.06 \\
1.44)\end{array}$ & $\begin{array}{l}1.42(1.21, \\
1.66)\end{array}$ & $<0.0001$ & \multirow[t]{2}{*}{0.02} \\
\hline No & reference & $\begin{array}{l}1.12(0.94 \\
1.34)\end{array}$ & $\begin{array}{l}1.28(1.08, \\
1.53)\end{array}$ & $\begin{array}{l}1.63(1.36, \\
1.96)\end{array}$ & $<0.0001$ & \\
\hline \multicolumn{7}{|l|}{ Diabetes } \\
\hline Yes & reference & $\begin{array}{l}1.07(0.86, \\
1.33)\end{array}$ & $\begin{array}{l}1.12(0.89, \\
1.4)\end{array}$ & $\begin{array}{l}1.17(0.93, \\
1.47)\end{array}$ & 0.18 & \multirow[t]{2}{*}{0.22} \\
\hline No & reference & $\begin{array}{l}1.02(0.90, \\
1.16)\end{array}$ & $\begin{array}{l}1.30(1.15 \\
1.48)\end{array}$ & $\begin{array}{l}1.71(1.49, \\
1.96)\end{array}$ & $<0.0001$ & \\
\hline \multicolumn{7}{|l|}{ Anemia } \\
\hline Yes & reference & $\begin{array}{l}1.09(0.70 \\
1.70)\end{array}$ & $\begin{array}{l}0.95(0.60 \\
1.53)\end{array}$ & $\begin{array}{l}0.94(0.59 \\
1.50)\end{array}$ & 0.69 & \multirow[t]{2}{*}{0.02} \\
\hline No & reference & $\begin{array}{l}1.09(0.97 \\
1.23)\end{array}$ & $\begin{array}{l}1.35(1.20 \\
1.51)\end{array}$ & $\begin{array}{l}1.60(1.41, \\
1.81)\end{array}$ & $<0.0001$ & \\
\hline \multicolumn{7}{|c|}{$\begin{array}{l}\text { a NAFLD, non-alcohol fatty liver disease; HRs, hazard ratios; } \mathrm{Cl} \text {, confidence interval; BMI, body mass } \\
\text { index; MET, metabolic equivalent; PA, physical activity. }\end{array}$} \\
\hline \multicolumn{7}{|c|}{$\begin{array}{l}\text { b Analysis by Cox proportional hazards regression model. Adjusted for age, sex, body mass index, } \\
\text { smoking status, alcohol drinking status, family history of diseases (hypertension, cardiovascular } \\
\text { disease, hyperlipidemia and diabetes), hypertension, hyperlipidemia, diabetes, anemia. }\end{array}$} \\
\hline \multicolumn{7}{|c|}{${ }^{\mathrm{c}} P$ for interaction was calculated using likelihood ratio test. } \\
\hline
\end{tabular}

The area under the ROC curve (AUC) values for serum ferritin levels were 0.61. Moreover, Evaluating the diagnostic performance of serum ferritin levels for NAFLD, the ROC yielded an optimal cut-off value of $55.9 \mathrm{mg} / \mathrm{dl}$, with a sensitivity and specificity of $68.5 \%$ and $48.7 \%$, respectively.

\section{Discussion}

In this prospective cohort study, we investigated the association between serum ferritin levels and the risk of incident NAFLD. The present results showed that serum ferritin levels were significantly and positively 
associated with the incidence of NAFLD after adjustment for multiple confounding factors among largescale adult population in China.

In the present analysis, multiple potentially confounding factors were adjusted. This study suggested that numerous factors (age, sex, BMI, WC, lipids, BP, FBG, smoking status, family history of some diseases) are associated with the incidence of NAFLD. Since studies have shown that age, sex, and BMI were major influence factors for the risk of NAFLD [19], we first adjusted for these three variables. Adjustment for age, sex, and BMI significantly affected the association between serum ferritin levels and incident NAFLD, leading us to conclude that age, sex, and BMI are major confounding factors. We subsequently adjusted for baseline ferritin levels, some chronic diseases [20], smoking status [21], alcohol drinking status [22], and genetic factors, such as a family history of CVD, hypertension, hyperlipidemia, and diabetes [23], which may influence the essential association between serum ferritin levels and the incidence of NAFLD. However, after these adjustments, a positive and significant association between serum ferritin levels and the risk of NAFLD remains.

Several cross-sectional and case-control studies have assessed the association between serum ferritin levels and the risk of NAFLD [24-27]. Recent a cross-sectional study found that serum ferritin positively associated with alanine aminotransferase (ALT) among subjects with overweight/obesity and ultrasound-confirmed liver steatosis [24]. Two cross-sectional studies also showed that higher serum ferritin was associated with a higher risk of NAFLD and elevated ALT among Chinese adults with normal weight $[25,26]$. In addition, a Korean study conducted on postmenopausal women found that the serum ferritin level was positively and independently associated with NAFLD [27]. Most cross-sectional studies reported a positive association between serum ferritin levels and the prevalence of NAFLD, however, cross-sectional study designs cannot be used to determine causal relationships because temporality is not known.

To the best of our knowledge, only one cohort study conducted in Korea assessed the association between serum ferritin levels and the incidence of NAFLD [12]. The study comprised 2,410 healthy Korean males who were aged 30 to 59 years old and found that the HR $(95 \% \mathrm{Cl})$ for incidence of NAFLD comparing the highest quartile of serum ferritin level to the lowest quartile was 1.41 (1.11-1.79) [12]. Our results were similar to the Korean study. Moreover, we also found a positive association among the total population and females.

Several possible mechanisms may explain the positive association between serum ferritin levels and incident NAFLD. Firstly, serum ferritin levels reflected body iron storage status. Previous experiments using animal models found that the mice exhibited significant increases in FFA levels and insulin resistance after feeding for 4 weeks on a high-fat, high-fructose diet to induce a hepatic iron overload model [8]. FFA levels and insulin resistance were risk factors for the development of NAFLD [28, 29]. Secondly, iron overload can promote the production of reactive oxygen species [30], which increase inflammation levels and oxidative stress, subsequently resulting in dysregulation of hepatic lipid 
metabolism and liver injury [31]. Finally, previous rat experiments found that iron overload caused liver mitochondrial dysfunction [9], which plays an important role in the development of NAFLD [32].

The main advantage of the present cohort study was the first cohort study to assess the association between serum ferritin levels and incident NAFLD in China. Other advantages included the study design, large sample size, and adjustment for multiple confounding factors. There are several limitations needed to be noted. First, although this study adjustment for lots of confounding factors, some unmeasured factors and residual confounding may influence the associations. Second, the present results only represent the study region. Further studies are needed to verify the results in other populations. Second, NAFLD was assessed by ultrasonography but not liver biopsy (the gold standard). However, a metaanalysis showed that liver ultrasonography is an accurate, reliable tool to detect moderate to severe fatty liver [33]. Due to its low cost, safety, and accessibility, ultrasound may be the imaging technique of choice for fatty liver screening in clinics and large sample size populations study.

\section{Conclusions}

The present study demonstrated that serum ferritin levels were positively associated with the incidence of NAFLD in a large-scale adult population. Further cohort studies are essential to confirm the findings in other populations.

\section{Declarations}

\section{Acknowledgments}

The authors gratefully acknowledge all the people that have made this study.

\section{Authors contributions}

Y.G., Q.L. and K.N. contributed to the study conception and design; Y.G., G.M., X.C., Q.Z., L.L., H.W., S.Z., S.S., X.W., J.Q., K.S. and K.N. contributed to data collection, assembly, analysis and interpretation of the data; Y.G., Q.L. and K.N. contributed to the manuscript drafting. All authors approved of the final version of the manuscript.

\section{Source of Funding}

This study was supported by grants from the National Natural Science Foundation of China (No. 91746205, 81872611, and 81673166), China.

\section{Conflicts of Interest}

All the authors declare that they have no competing interests.

\section{Ethics approval}


The study was approved by The Institutional Review Board of Tianjin Medical University, Tianjin, China.

\section{Consent to participate}

All participants provided written informed consent.

\section{References}

1. Bellentani S, Scaglioni F, Marino M, Bedogni G. Epidemiology of non-alcoholic fatty liver disease. Dig Dis. 2010;28(1):155-61.

2. Neuschwander-Tetri BA. Non-alcoholic fatty liver disease. BMC Med. 2017;15(1):45.

3. Younossi ZM, Koenig AB, Abdelatif D, Fazel Y, Henry L, Wymer M. Global epidemiology of nonalcoholic fatty liver disease-Meta-analytic assessment of prevalence, incidence, and outcomes. Hepatology. 2016;64(1):73-84.

4. Younossi Z, Tacke F, Arrese M, Chander Sharma B, Mostafa I, Bugianesi E, et al. Global Perspectives on Nonalcoholic Fatty Liver Disease and Nonalcoholic Steatohepatitis. Hepatology. 2019;69(6):2672-82.

5. Zhou F, Zhou J, Wang W, Zhang XJ, Ji YX, Zhang P, et al. Unexpected Rapid Increase in the Burden of NAFLD in China From 2008 to 2018: A Systematic Review and Meta-Analysis. Hepatology. 2019;70(4):1119-33.

6. Adams LA, Anstee QM, Tilg H, Targher G. Non-alcoholic fatty liver disease and its relationship with cardiovascular disease and other extrahepatic diseases. Gut. 2017;66(6):1138-53.

7. Wang W, Knovich MA, Coffman LG, Torti FM, Torti SV. Serum ferritin: Past, present and future. Biochim Biophys Acta. 2010;1800(8):760-9.

8. Tsuchiya H, Ebata Y, Sakabe T, Hama S, Kogure K, Shiota G. High-fat, high-fructose diet induces hepatic iron overload via a hepcidin-independent mechanism prior to the onset of liver steatosis and insulin resistance in mice. Metabolism. 2013;62(1):62-9.

9. Ceccarelli D, Gallesi D, Giovannini F, Ferrali M, Masini A. Relationship between free iron level and rat liver mitochondrial dysfunction in experimental dietary iron overload. Biochem Biophys Res Commun. 1995;209(1):53-9.

10. Lee HJ, Choi JS, Lee HJ, Kim WH, Park SI, Song J. Effect of excess iron on oxidative stress and gluconeogenesis through hepcidin during mitochondrial dysfunction. J Nutr Biochem. 2015;26(12):1414-23.

11. Friedman SL, Neuschwander-Tetri BA, Rinella M, Sanyal AJ. Mechanisms of NAFLD development and therapeutic strategies. Nat Med. 2018;24(7):908-22.

12. Kim CW, Chang Y, Sung E, Shin H, Ryu S. Serum ferritin levels predict incident non-alcoholic fatty liver disease in healthy Korean men. Metabolism. 2012;61(8):1182-8.

13. Gu Y, Li H, Bao X, Zhang Q, Liu L, Meng G, et al. The Relationship Between Thyroid Function and the Prevalence of Type 2 Diabetes Mellitus in Euthyroid Subjects. J Clin Endocrinol Metab. 
2017;102(2):434-42.

14. Alcoholic Liver Disease CSoHCMA, Fatty Liver Expert Committee CMDA. [Guidelines of prevention and treatment for nonalcoholic fatty liver disease: a 2018 update]. Zhonghua Gan Zang Bing Za Zhi. 2018;26(3):195-203.

15. American Diabetes A. Standards of medical care in diabetes--2013. Diabetes Care. 2013;36(Suppl 1):11-66.

16. Expert Panel on Detection E. Treatment of High Blood Cholesterol in A. Executive Summary of The Third Report of The National Cholesterol Education Program (NCEP) Expert Panel on Detection, Evaluation, And Treatment of High Blood Cholesterol In Adults (Adult Treatment Panel III). JAMA. 2001;285(19):2486-97.

17. WHO. Haemoglobin concentrations for the diagnosis of anaemia and assessment of severity. Vitamin and Mineral Nutrition Information System. Geneva, World Health Organization, 2011 WHO/NMH/NHD/MNM/11.1 (http://www.who.int/vmnis/indicators/haemoglobin.pdf, accessed May 2021).

18. Chobanian AV, Bakris GL, Black HR, Cushman WC, Green LA, Izzo JL Jr, et al. The Seventh Report of the Joint National Committee on Prevention, Detection, Evaluation, and Treatment of High Blood Pressure: the JNC 7 report. JAMA. 2003;289(19):2560-72.

19. Tang Z, Pham M, Hao Y, Wang F, Patel D, Jean-Baptiste L, et al. Sex, Age, and BMI Modulate the Association of Physical Examinations and Blood Biochemistry Parameters and NAFLD: A Retrospective Study on 1994 Cases Observed at Shuguang Hospital, China. Biomed Res Int. 2019;2019:1246518.

20. Lonardo A, Nascimbeni F, Mantovani A, Targher G. Hypertension, diabetes, atherosclerosis and NASH: Cause or consequence? J Hepatol. 2018;68(2):335-52.

21. Jung HS, Chang Y, Kwon MJ, Sung E, Yun KE, Cho YK, et al. Smoking and the Risk of Non-Alcoholic Fatty Liver Disease: A Cohort Study. Am J Gastroenterol. 2019;114(3):453-63.

22. Sogabe $M$, Okahisa T, Nakagawa T, Fukuno H, Nakasono M, Tomonari T, et al. Influence of light alcohol consumption on lifestyle-related diseases: a predictor of fatty liver with liver enzyme elevation in Japanese females with metabolic syndrome. BMC Gastroenterol. 2016;16:17.

23. Bhadoria AS, Kedarisetty CK, Bihari C, Kumar G, Jindal A, Bhardwaj A, et al. Impact of family history of metabolic traits on severity of non-alcoholic steatohepatitis related cirrhosis: A cross-sectional study. Liver Int. 2017;37(9):1397-404.

24. Galarregui C, Marin-Alejandre BA, Perez-Diaz-Del-Campo N, Cantero I, Monreal JI, Elorz M, et al. Predictive Value of Serum Ferritin in Combination with Alanine Aminotransferase and Glucose Levels for Noninvasive Assessment of NAFLD: Fatty Liver in Obesity (FLiO) Study. Diagnostics (Basel). 2020;10(11).

25. You G, Ding J, Shen J, Wang Y, Sun Y. Association between serum ferritin and non-alcoholic fatty liver disease among middle-aged and elderly Chinese with normal weight. Asia Pac J Clin Nutr. 2019;28(4):747-53. 
26. Yao J, Dai Y, Zhang J, Zhang X, Zheng R. Association between Serum Ferritin Level and Nonalcoholic Fatty Liver Disease in a Non-Obese Chinese Population: a Cross-Sectional Study. Clin Lab. 2019;65(6).

27. Kim HB, Lee HS, Lee YJ. Association of serum ferritin levels with non-alcoholic fatty liver disease in postmenopausal women. Climacteric. 2018;21(5):509-14.

28. Finck BN. Targeting Metabolism, Insulin Resistance, and Diabetes to Treat Nonalcoholic Steatohepatitis. Diabetes. 2018;67(12):2485-93.

29. Gambino R, Bugianesi E, Rosso C, Mezzabotta L, Pinach S, Alemanno N, et al. Different Serum Free Fatty Acid Profiles in NAFLD Subjects and Healthy Controls after Oral Fat Load. Int J Mol Sci. 2016;17(4):479.

30. Nakamura T, Naguro I, Ichijo H. Iron homeostasis and iron-regulated ROS in cell death, senescence and human diseases. Biochim Biophys Acta Gen Subj. 2019;1863(9):1398-409.

31. Cobbina E, Akhlaghi F. Non-alcoholic fatty liver disease (NAFLD) - pathogenesis, classification, and effect on drug metabolizing enzymes and transporters. Drug Metab Rev. 2017;49(2):197-211.

32. Mansouri A, Gattolliat $\mathrm{CH}$, Asselah T. Mitochondrial Dysfunction and Signaling in Chronic Liver Diseases. Gastroenterology. 2018;155(3):629-47.

33. Hernaez R, Lazo M, Bonekamp S, Kamel I, Brancati FL, Guallar E, et al. Diagnostic accuracy and reliability of ultrasonography for the detection of fatty liver: a meta-analysis. Hepatology. 2011;54(3):1082-90.

\section{Figures}




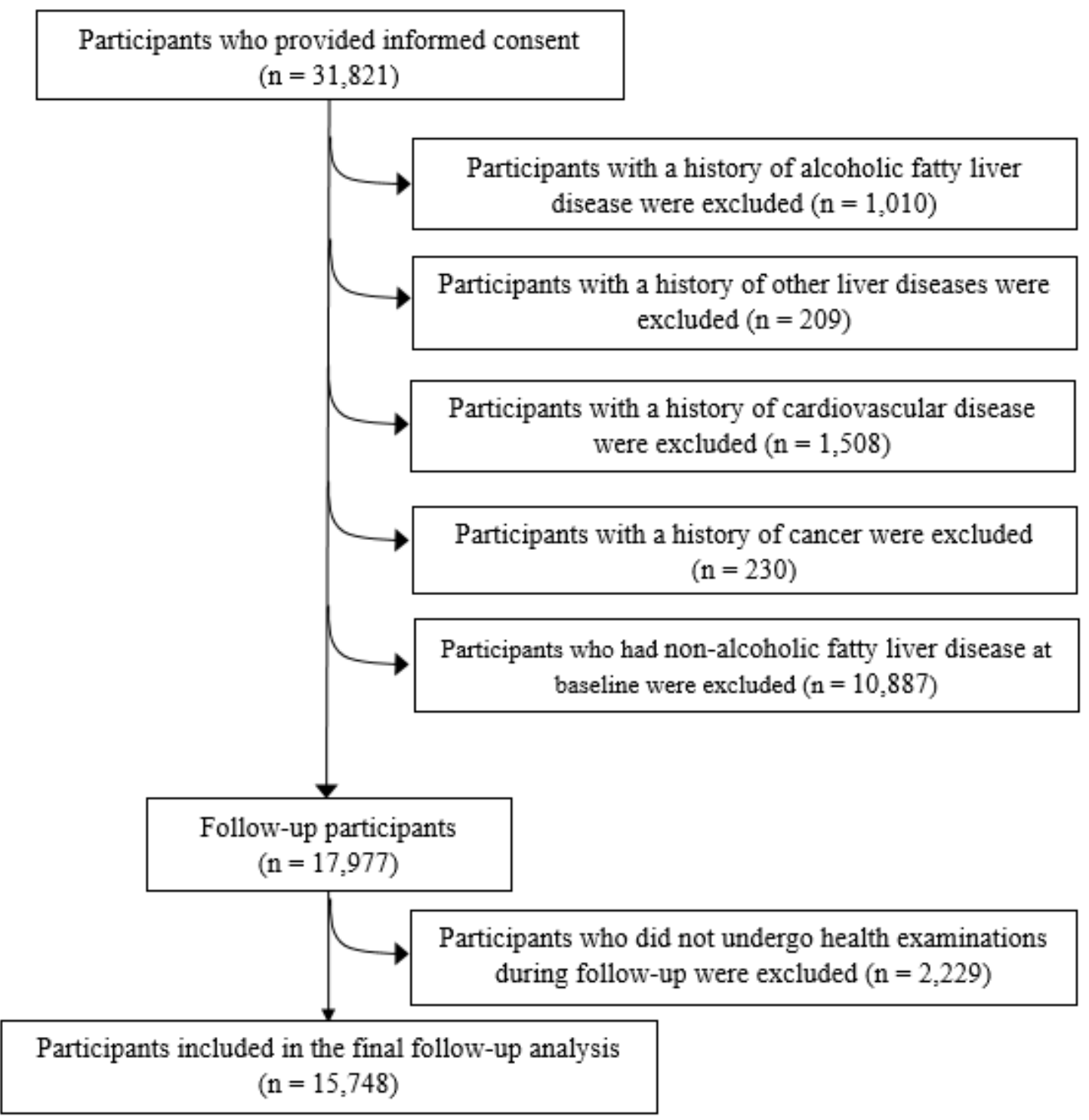

Figure 1

Flow chart of population selection

\section{Supplementary Files}

This is a list of supplementary files associated with this preprint. Click to download.

- SupplementalTable1.docx 